\title{
Exercise therapy versus arthroscopic partial meniscectomy for degenerative meniscal tear in middle aged patients: randomised controlled trial with two year follow-up
}

\author{
Nina Jullum Kise, ${ }^{1}$ May Arna Risberg, ,3,3 Silje Stensrud, ${ }^{2}$ Jonas Ranstam, ${ }^{5}$ Lars Engebretsen, 3,6,7 \\ Ewa M Roos ${ }^{8}$
}

${ }^{1}$ Department of Orthopaedic

Surgery, Martina Hansens

Hospital, PO box 823, N-1306

Sandvika, Norway

${ }^{2}$ Norwegian Research Centre for Active Rehabilitation, Oslo,

Norway

${ }^{3}$ Division of Orthopaedic

Surgery, Oslo University

Hospital, Norway

${ }^{4}$ Department of Sports

Medicine, Norwegian School of

Sport Sciences, Oslo, Norway

${ }^{5}$ Department of Orthopaedics,

Clinical Sciences Lund, Lund

University, Sweden

${ }^{6}$ Faculty of Medicine, University in Oslo

${ }^{7}$ Oslo Sports Trauma Research

Centre, Norwegian School of

Sport Sciences, Oslo, Norway

${ }^{8}$ Research Unit for

Musculoskeletal Function and

Physiotherapy, Institute of

Sports Science and Clinical

Biomechanics, University of

Southern Denmark, Odense,

Denmark

Correspondence to:

N J Kisenina.kise@mhh.no

Additional material is published

online only. To view please visit

the journal online.

Cite this as: BMJ 2016;354:i3740 http://dx.doi.org/10.1136/bmj.i3740

Accepted: 26 June 2016

\section{ABSTRACT}

OBJECTIVE

To determine if exercise therapy is superior to arthroscopic partial meniscectomy for knee function in middle aged patients with degenerative meniscal tears.

DESIGN

Randomised controlled superiority trial.

SETTING

Orthopaedic departments at two public hospitals and two physiotherapy clinics in Norway.

PARTICIPANTS

140 adults, mean age 49.5 years (range 35.7-59.9), with degenerative medial meniscal tear verified by magnetic resonance imaging. $96 \%$ had no definitive radiographic evidence of osteoarthritis.

\section{INTERVENTIONS}

12 week supervised exercise therapy alone or arthroscopic partial meniscectomy alone.

\section{MAIN OUTCOME MEASURES}

Intention to treat analysis of between group difference in change in knee injury and osteoarthritis outcome score $\left(\mathrm{KOOS}_{4}\right)$, defined a priori as the mean score for four of five KOOS subscale scores (pain, other symptoms, function in sport and recreation, and knee related quality of life) from baseline to two year follow-up and change in thigh muscle strength from baseline to three months.

\section{RESULTS}

No clinically relevant difference was found between the two groups in change in $\mathrm{KOOS}_{4}$ at two years $(0.9$ points, $95 \%$ confidence interval -4.3 to $6.1 ; \mathrm{P}=0.72$ ).

\section{WHAT IS ALREADY KNOWN ON THIS TOPIC}

Interventions that include knee arthroscopy are associated with a small benefit and with harms; the small benefit is inconsequential and of short duration

Most previous studies were performed in patients with radiographic evidence of knee osteoarthritis

Those studies were designed to study the additional benefit from knee arthroscopy, and the exercise programmes were often of insufficient quality

\section{WHAT THIS STUDY ADDS}

Exercise therapy and knee arthroscopy were similarly effective for pain relief and other patient reported outcomes in a younger, more active population with a lower body mass index than previously studied

Exercise therapy resulted in better thigh muscle strength than surgery

Supervised exercise therapy should be considered as a treatment option for patients with pain and degenerative meniscal tears verified by magnetic resonance imaging, and without radiographic signs of osteoarthritis

At three months, muscle strength had improved in the exercise group $(\mathrm{P} \leq 0.004)$. No serious adverse events occurred in either group during the two year follow-up. $19 \%$ of the participants allocated to exercise therapy crossed over to surgery during the two year follow-up, with no additional benefit.

\section{CONCLUSION}

The observed difference in treatment effect was minute after two years of follow-up, and the trial's inferential uncertainty was sufficiently small to exclude clinically relevant differences. Exercise therapy showed positive effects over surgery in improving thigh muscle strength, at least in the short term. Our results should encourage clinicians and middle aged patients with degenerative meniscal tear and no definitive radiographic evidence of osteoarthritis to consider supervised exercise therapy as a treatment option.

TRIAL REGISTRATION

www.clinicaltrials.gov (NCT01002794).

\section{Introduction}

In the Western world, as many as 300 in 100000 people undergo arthroscopic partial meniscectomy annually. ${ }^{1-3}$ In Denmark, the surgery rate doubled from 2000 to $2011,{ }^{4}$ with three out of four patients aged more than 35 years. ${ }^{4}$ In these patients, most meniscal tears are degenerative and might be regarded as the first sign of osteoarthritis. ${ }^{56}$

In a recent meta-analysis, the authors concluded that a small but inconsequential benefit is seen from treatment interventions that involve arthroscopy. ${ }^{7}$ This small effect is of short duration and absent one year after surgery. ${ }^{7}$ Only one ${ }^{8}$ in five randomised controlled trials ${ }^{8-13}$ found greater pain relief one year after partial meniscectomy compared with non-surgical treatment. ${ }^{8}$ Short term and long term follow-up studies have shown that exercise therapy improves function and activity level in patients with degenerative meniscal tears, regardless of whether they have surgery. ${ }^{9-11} 13$

Only one small pilot study ( $\mathrm{n}=17$ ) compared the effect of surgery alone with exercise alone. ${ }^{14}$ Of the five other published randomised controlled trials, ${ }^{8-13}$ four $^{8-1113}$ were designed to study the effect of surgery in addition to exercise therapy, and the remaining study ${ }^{12}$ compared meniscectomy to sham surgery. Considering the large amount of surgery performed worldwide, and the inconsequential short term additional pain relief seen from surgery in addition to exercise, randomised controlled trials are needed to explore the comparative treatment effect of partial meniscectomy alone with supervised exercise therapy alone. Furthermore, only 
two of the five published randomised controlled trials included patients with no definitive radiographic evidence of osteoarthritis. ${ }^{1213}$ The aim of this study was to determine if exercise therapy is superior to arthroscopic surgery for knee function in middle aged patients with degenerative meniscal tears verified by magnetic resonance imaging.

\section{Methods}

Trial design

In this randomised controlled trial with two parallel intervention groups (1:1 ratio) we compared exercise therapy alone with arthroscopic partial meniscectomy alone. Follow-up assessments were performed at three, 12, and 24 months, with muscle strength at three months and patient reported outcomes at the two year follow-up as the primary end points. Whereas data at three and 12 months were collected during clinic visits, the follow-up at two years was conducted by post, and we only collected data on patient reported outcomes.

\section{Deviations from trial registration}

Owing to financial and logistical constraints, we conducted tests on muscle strength and performance at 12 months instead of the 24 months stated in the trial registration. Muscle strength at three months for the first 82 patients has been previously reported. ${ }^{15}$ A recent meta-analysis of surgically treated patients found that weakness of the extensor muscle already existed in legs before surgery, and this remained largely for at least four years after surgery. ${ }^{16}$ Considering these results, we did not think that obtaining muscle function at 24 months in addition to at 12 months would have changed the interpretation of our results. Radiographs will be obtained at the five year follow-up and are therefore unavailable for this two year report. Quality of life (EQ5D) was not analysed at two years but will be reported as intended at five years.

\section{Participants}

Between October 2009 and September 2012, we recruited participants from the orthopaedic departments at Oslo University Hospital (October 2009-April 2011) and Martina Hansens Hospital (May 2011-September 2012) in Norway. All patients provided informed written consent before participation.

Inclusion criteria were age 35-60 years; unilateral knee pain for more than two months without a major trauma (defined as sudden onset of knee pain resulting from a single physical impact event); medial degenerative meniscal tear verified by magnetic resonance imaging; and, at most, radiographic changes equivalent to grade 2 according to the Kellgren-Lawrence classification. ${ }^{17}$ Standing posterior-anterior radiographs were taken in a fixed flexion position, using a Synaflexer (Synarc, Newark, CA) frame. ${ }^{18}$ We defined a degenerative meniscal tear as an intrameniscal linear magnetic resonance imaging signal penetrating one or both surfaces of the meniscus. ${ }^{19}$ Furthermore, the patients had to be eligible for arthroscopy, be able to participate in exercise therapy, and understand Norwegian. One of two orthopaedic surgeons confirmed eligibility for surgery based on the patient's history, physical examination, and findings on magnetic resonance imaging. Exclusion criteria were acute trauma, locked knee, ligament injury, and knee surgery in the index knee during the previous two years.

\section{Interventions}

The exercise therapy intervention was carried out at one of two clinics (Norwegian Sports Medicine Clinic and Gnist Trening og Helse AS), using the same protocol and started as soon as possible after randomisation-or later if preferred by the participant. The exercise therapy programme, outlined in supplementary figure S1 and previously described in detail, ${ }^{20}$ consisted of progressive neuromuscular and strength exercises over 12 weeks, performed during a minimum of two and a maximum of three sessions each week (24-36 sessions).

The participants filled in exercise diaries, and we assessed compliance with exercise as the total number of exercise sessions completed out of 24 sessions. Excellent compliance was predefined as participation in 24 or more sessions $(100 \%)$, satisfactory compliance as $19-23$ sessions ( $80-100 \%)$, and poor compliance as 18 or fewer sessions $(<80 \%)$. In the per protocol analysis, we defined completing 18 or fewer sessions as not following the protocol. Likewise, if participants in the meniscectomy group received physiotherapist instructed exercise therapy postoperatively of adequate quality for at least 18 sessions, they were defined as not following the protocol.

Arthroscopic surgery was performed as soon as possible after randomisation, depending on waiting lists and participant preference. The arthroscopic intervention was similar in both hospitals, performed as standard operations for arthroscopic partial meniscectomy, and the participants followed normal preoperative, perioperative, and postoperative routines. Six orthopaedic surgeons with at least 10 years of clinical experience performed the operations. One surgeon performed 39 (61\%) operations, and the other five surgeons performed 1-15 operations each. The participants were discharged from hospital on the day of surgery and were advised to use two crutches postoperatively until gait normalised and no swelling or discomfort occurred during weight bearing. Before hospital discharge the participants were given written and oral instructions for simple home exercises, aimed at regaining knee range of motion and reducing swelling. They were encouraged to perform the exercises two to four times daily (see supplementary figure S2a-d for written instructions).

Surgery was performed with the participant under general anaesthesia, with or without thigh tourniquet, antibiotic prophylaxis, or antithrombotic prophylaxis. Arthroscopes with 30 degree optics and standard arthroscopic instruments were used. Ringer acetate was used for lavage. Normal procedure involved two portals: anteromedial and anterolateral, and if required, additional portals were made and a lavage cannula was inserted laterally in the cranial recess. A diagnostic procedure including evaluation of 
additional injuries (ligaments, cartilage) preceded systematic probing of both menisci, and, finally, all unstable meniscal tissue was resected.

\section{Primary outcomes}

Our two primary endpoints were patient reported knee function at two years and thigh muscle strength at three months. The primary patient reported endpoint was change from baseline to two years in $\mathrm{KOOS}_{4}$, defined as the average score for four of the five knee injury and osteoarthritis outcome score (KOOS) subscale scores covering pain, other symptoms, function in sport and recreation, and knee related quality of life. KOOS is reliable and has content validity for patients with meniscal tears and osteoarthritis. ${ }^{21} 22$ It consists of 42 items scored from 0-4 on a Likert scale. Subscale scores are calculated separately and transformed to a scale from 0 (worst) to 100 (best). A priori, a clinically relevant difference of 10 points guided the sample size calculation. To better guide clinical interpretation, we calculated study specific and subscale specific cut-offs post hoc by subtracting the mean KOOS subscale score for those reporting to have "unchanged" knee function from those reporting "better" knee function at two years, on a five point global rating scale (much better, better, unchanged, worse, or much worse)..$^{23}$

Experienced physiotherapists used detailed test protocols to collect data on muscle strength. A Biodex 6000 dynamometer was used to test the strength of quadriceps and hamstrings concentric isokinetic muscle. The outcomes were peak torque and total work for both knee extension and knee flexion at 60 degrees per second. The reliability for isokinetic muscle tests is satisfactory. ${ }^{24}$

\section{Secondary outcomes}

Secondary patient reported outcomes were the five KOOS subscales and the physical component summary and mental component summary of the short form 36 item (SF-36). ${ }^{25}$ Secondary objective outcomes were thigh muscle strength and lower extremity performance test results.

We used three reliable and valid performance tests $^{26-28}$ to evaluate lower extremity function: the one leg hop test for distance (measuring length in centimetres), the $6 \mathrm{~m}$ timed hop test (measuring time in seconds), and the knee bends test (measuring maximum number in 30 seconds). These test procedures have been described previously. ${ }^{29}$ The test protocol included a 10 minute warm-up on a stationary bicycle, followed by the muscle strength and lower extremity performance tests. The first leg to be tested was determined by randomisation, and the same order was applied at the follow-up assessments. Tests of thigh muscle strength and lower extremity performance were conducted at baseline and at three and 12 month follow-ups.

Adverse events and serious adverse events were recorded and categorised into index knee or other sites. At all follow-up assessments we asked the participants about potential adverse events, and at the two year follow-up we checked the medical charts from the participating hospitals. We defined any situations where participants sought healthcare as adverse events, with death, cardiovascular or gastrointestinal events, deep vein thrombosis, pulmonary embolism, and systemic or local infection categorised as serious adverse events. We categorised knee symptoms such as pain, swelling, instability, and decreased range of motion as adverse events only if the participant sought treatment. The participants were encouraged to contact the participating hospitals for additional clinic visits with the orthopaedic surgeon if needed.

\section{Sample size}

The sample size calculation was based on the change in $\mathrm{KOOS}_{4}$ from baseline to two year follow-up. To detect a 10 point difference with a standard deviation of 15 , with a level of power of $90 \%$, level of significance of 0.05 , and an estimated 15\% dropout rate at two years, we determined that we would need 56 participants in each group. To allow for a $20 \%$ crossover rate, we randomised 140 participants.

\section{Randomisation}

Participants contributed baseline data before they were randomly allocated to one of two parallel intervention groups, treated with either arthroscopic partial meniscectomy or exercise therapy. A statistician at Oslo University Hospital determined the computer generated randomisation sequence, stratified by sex in blocks of eight, and these were concealed from the surgeons who enrolled and assessed the participants. The allocations were kept in sequentially numbered opaque envelopes that were opened by the participants after enrolment.

\section{Blinding}

The test assessors were blinded to group allocation, and long pants or neoprene sleeves were worn by participants over both knees to hide possible surgical scars and preserve blinding of group allocation. The statistician was blinded to group allocation during the analysis.

\section{Statistical analysis}

The statistical analyses were performed according to the a priori published statistical analysis plan (http://static.sdu.dk/mediafiles//5/E/5/\%7B5 E568A02-9475-4127-9B6C-0ADA7235DF77\%7DSAP\%20 OMEX\%2020.03.15.pdf). All participants assigned to treatment were included in the intention to treat analysis. Between group comparison of the primary patient reported endpoint, change in $\mathrm{KOOS}_{4}$ from baseline to two year follow-up, was made with the use of a repeated measures mixed model, stratified by sex and study site and with adjustment for baseline imbalance of $\mathrm{KOOS}_{4}$ scores.

We analysed between group comparisons of the change from baseline to the 12 month and 24 month follow-up assessments on secondary outcome measures (the five KOOS subscales, SF-36 physical component summary and mental component summary, and performance tests) by intention to treat, similar to the primary 
outcome measures. In addition to the intention to treat analysis, we performed per protocol and as treated analyses. We present the KOOS, SF-36, and performance scores as means with $95 \%$ confidence intervals.

IBM SPSS Statistics version 22 (IBM, Armonk, NY) was used for descriptive analysis of baseline data, and Stata v14 (Stata 2015, College Station, TX) was used for analysis of outcomes at three, 12, and 24 months. The mixed model analysis was based on the assumption that the covariance structure had a compound symmetrythat is, that the total variation could be partitioned into two components representing variation between participants and within participants. The model was fitted using the mixed-command, and we used Satterthwaite's method to calculate the degrees of freedom. ${ }^{30}$

\section{Patient involvement}

No patients were involved in setting the research question or the outcome measures, nor were they involved in developing plans for recruitment, design, or implementation of the study. No patients were asked to advise on interpretation or writing up of results. When the results of this randomised controlled trial are published, information will be conveyed to the participants in lay language in a pamphlet distributed by email.

\section{Results}

Out of 341 patients assessed for eligibility, 226 were eligible and $140(41 \%)$ were randomised to the two treatment groups, each with 70 participants. Questionnaires were completed by 129 participants (92\%) at three and 12 months and 126 (90\%) at two years (fig 1).

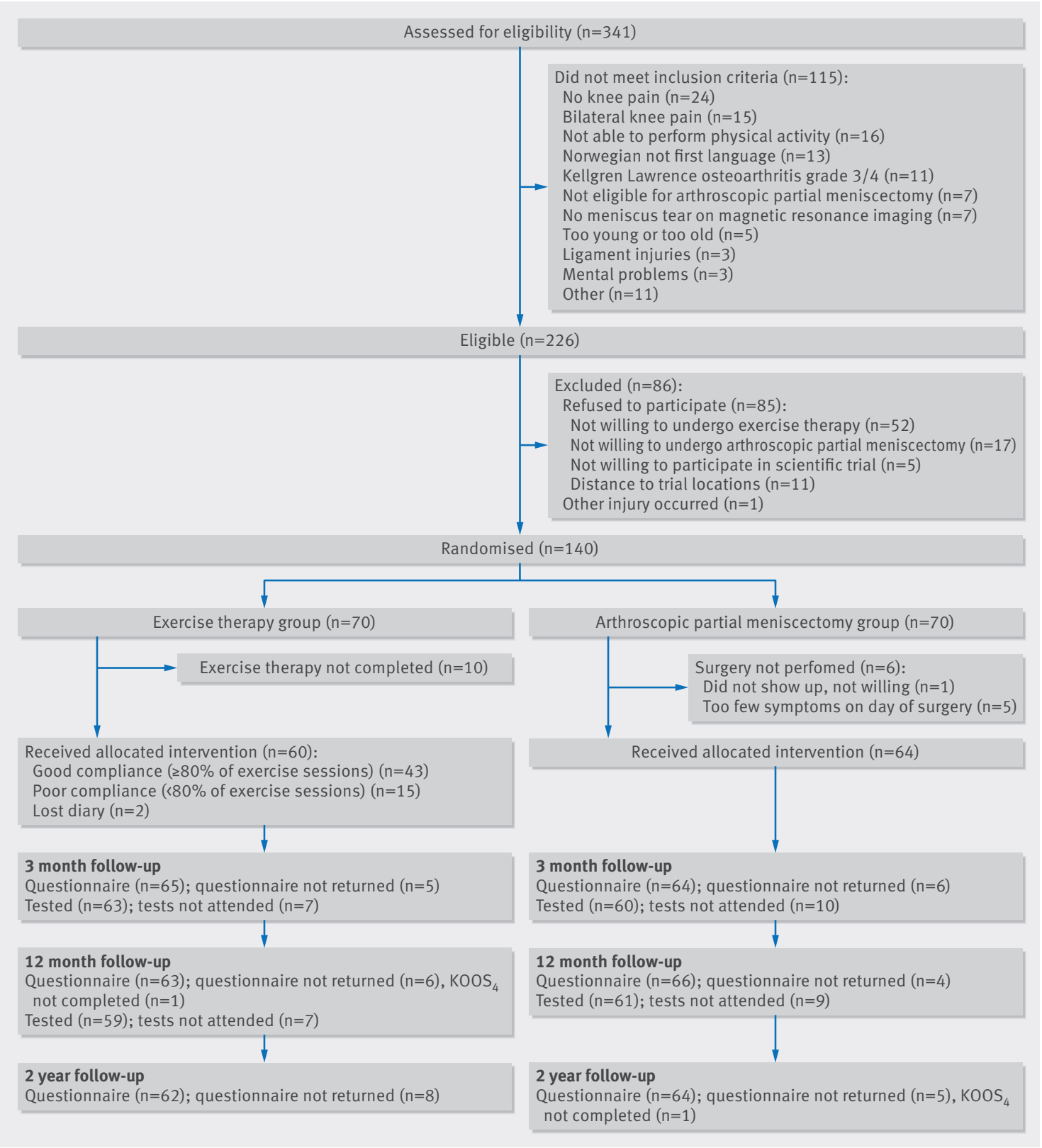

Fig 1 | Flow chart of participants through study. $\mathrm{KOOS}_{4}=$ mean of knee injury and osteoarthritis outcome score subscales for pain, other symptoms, function in sport and recreation, and knee related quality of life 
In the exercise group, 43 out of $70(61 \%)$ participants completed the exercise therapy programme with satisfactory (17 participants) or excellent (26 participants) compliance. These participants on average completed 25 exercise sessions (median 25, range 19-36). Fifteen participants had poor compliance, 10 declined exercise therapy, and two had lost their exercise diaries. In the arthroscopic partial meniscectomy group, six

\begin{tabular}{|c|c|c|}
\hline Characteristics & Exercise group & Meniscectomy group \\
\hline Demographics: & $n=70$ & $n=70$ \\
\hline No (\%) men & $43(61)$ & $43(61)$ \\
\hline No (\%) right knee & $41(59)$ & $41(59)$ \\
\hline Age (years) & $50.2(6.2)$ & $48.9(6.1)$ \\
\hline Body mass index (weight $(\mathrm{kg}) /\left(\right.$ height $\left.(\mathrm{m})^{2}\right)$ ) & $26.4(4.3)$ & $26.0(3.7)$ \\
\hline No $(\%)$ smokers & $3(4.2)$ & $10(14.3)$ \\
\hline No (\%) use analgesics daily & $3(4.2)$ & $3(4.2)$ \\
\hline No (\%) primary school education only & $3(4.2)$ & $2(2.9)$ \\
\hline No (\%) education at university level & $37(53)$ & $36(51)$ \\
\hline Severity of radiographic knee osteoarthritis*: & $n=70$ & $n=70$ \\
\hline Grade 0 & $49(70)$ & $51(73)$ \\
\hline Grade 1 & $18(26)$ & $16(23)$ \\
\hline Grade 2 & $2(3)$ & $3(4)$ \\
\hline Grade 3 & $1(1)$ & 0 \\
\hline Magnetic resonance imagingt: & $n=69$ & $\mathrm{n}=70$ \\
\hline \multicolumn{3}{|l|}{ Meniscal degeneration $\ddagger$} \\
\hline No $(\%)$ grade $1-2$ & $6(9)$ & $6(9)$ \\
\hline No (\%) grade $3 a-3 b$ & $63(91)$ & $64(91)$ \\
\hline \multicolumn{3}{|l|}{ Meniscal extrusion§ } \\
\hline No (\%) no extrusion & $24(35)$ & $35(50)$ \\
\hline No (\%) extrusion & $45(65)$ & $35(50)$ \\
\hline Pain: & $\mathrm{n}=70$ & $\mathrm{n}=69$ \\
\hline Duration (months) & $17.3(21.5)$ & $12.0(15.7)$ \\
\hline Knee function: & $n=67$ & $n=63$ \\
\hline Visual analogue scale (0-100, worse to best) & $57.9(21.5)$ & $63.8(18.9)$ \\
\hline KOOS scores (0-100, worst to best): & $\mathrm{n}=70$ & $\mathrm{n}=70$ \\
\hline $\mathrm{KOOS}_{4}$ & $54.3(18.2)$ & $59.6(13.8)$ \\
\hline Pain & $63.4(20.8)$ & $67.6(14.9)$ \\
\hline Symptoms & $69.8(16.7)$ & $77.4(14.6)$ \\
\hline Activities of daily living & $75.0(21.5)$ & $79.6(16.1)$ \\
\hline Function in sport and recreation & $44.0(25.8)$ & $47.8(23.4)$ \\
\hline Knee related quality of life & $40.0(17.5)$ & $45.6(15.5)$ \\
\hline SF-36 points (0-100, worst to best): & $\mathrm{n}=70$ & $\mathrm{n}=70$ \\
\hline Physical component summary & $45.4(8.4)$ & $47.4(6.1)$ \\
\hline Mental component summary & $55.0(9.2)$ & $56.0(6.3)$ \\
\hline Muscle strength (higher is better): & $\mathrm{n}=70$ & $\mathrm{n}=70$ \\
\hline Peak torque extension $(\mathrm{Nm})$ & $157.5(48.7)$ & $163.1(53.2)$ \\
\hline Total work extension (J) & $772.9(245.1)$ & $790.8(254.8)$ \\
\hline Peak torque flexion $(\mathrm{Nm})$ & $81.9(27.2)$ & $88.5(25.7)$ \\
\hline Total work flexion (J) & $448.3(187.8)$ & $492.9(158.7)$ \\
\hline Performance tests: & $n=69$ & $n=69$ \\
\hline One leg hop test (cm) (higher is better) & $76.6(32.8)$ & $83.2(35.5)$ \\
\hline $6 \mathrm{~m}$ timed hop test (sec) (lower is better) & $3.1(1.7)$ & $2.7(1.2)$ \\
\hline Knee bends 30 sec test (No) (higher is better) & $28.2(10.6) 9$ & $29.3(10.6)$ \\
\hline \multicolumn{3}{|c|}{$\begin{array}{l}\text { KOOS=knee injury and osteoarthritis outcome score; SF-36=36 item short form; Nm=Newtonmetre; J=Joule. } \\
\text { *Kellgren-Lawrence classification. } \\
\text { tAlthough inclusion was based on clinical readings of baseline magnetic resonance images by several } \\
\text { radiologists and orthopaedic surgeons, the data presented here originate from post hoc reading by one } \\
\text { radiologist blinded to group allocation and study outcome. } \\
\text { fGraded according to Crues et al.19 } \\
\text { \$Evaluated on coronal sequence images, with largest tibial spine volume, defined as meniscal subluxation } \\
\text { crossing a vertical line on the medial margin of tibia without osteophytes. } \\
\uparrow \mathrm{n}=70 \text { participants. }\end{array}$} \\
\hline
\end{tabular}

participants out of 70 (9\%) did not undergo surgery, owing to personal preference (one participant) or too few knee symptoms on the day of surgery (five participants) (fig 1).

In the intention to treat analyses, the participants were included as randomised. Those who did not complete the assigned treatments were excluded from the per protocol analysis (see supplementary figure S3).

Thirteen out of 70 participants (19\%) in the exercise group crossed over to receive surgical treatment between three and 16 months (mean 7.7 months) after inclusion. Of these, approximately half had completed at least 19 exercise sessions. Participants who crossed over to surgery were analysed in the meniscectomy group in the as treated analysis (see supplementary figure S3).

Five participants in the meniscectomy group received passive postoperative physiotherapy (median 2 sessions, range 1-3), but none crossed over to exercise therapy.

Owing to persistent knee pain and catching of the knee, two participants (3\%) in the meniscectomy group were reoperated on at 12 and 15 months, respectively, and one participant who had crossed over underwent another operation six months after the primary operation. One participant in the meniscectomy group and one participant who crossed over from the exercise to meniscectomy group (both with Kellgren-Lawrence grade 1 osteoarthritis) underwent osteotomy at six and 16 months, respectively, after the index surgery, owing to increasing pain and further impaired knee function, which was related to osteoarthritis as diagnosed by the surgeon who followed them clinically. A third participant, in the meniscectomy group (Kellgren-Lawrence grade 1 osteoarthritis), was given a diagnosis of osteoarthritis and treated with passive physiotherapy and non-steroidal anti-inflammatory drugs. One participant from each group underwent arthroscopic partial meniscectomy of the contralateral knee at six months and four months, respectively.

\section{Baseline data}

Table 1 presents the baseline characteristics of the participants.

On an extra inspection of the radiographs, one participant allocated to the exercise group was found to have Kellgren-Lawrence grade 3 osteoarthritis, which was an exclusion criterion. This participant was thus unintentionally included, but had fulfilled all the follow-up assessments and was retained in all appropriate analyses because the results in a sensitivity analysis excluding this participant did not differ.

\section{Primary outcomes}

The study specific and subscale specific cut-off for a clinically relevant difference between groups in $\mathrm{KOOS}_{4}$ was 10.1. In the intention to treat analysis, there was no clinically relevant difference in change between groups from baseline to two year follow-up in $\mathrm{KOOS}_{4}$ score (0.9 points, 95\% confidence interval -4.3 to $6.1 ; \mathrm{P}=0.72$ ) after adjustment for baseline imbalance and randomisation stratification factors. The mean improvements were 25.3 


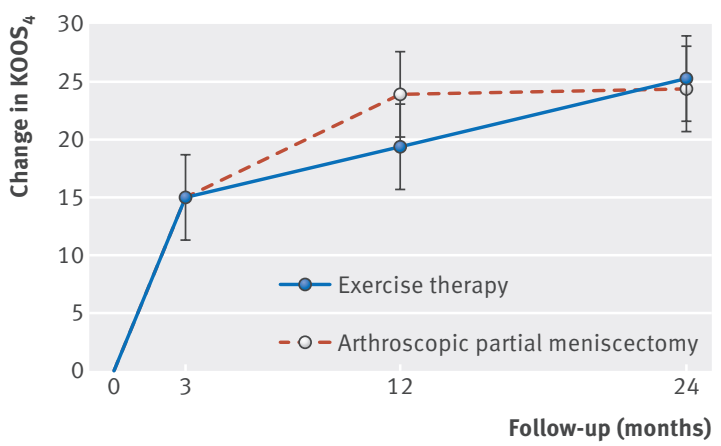

Fig 2 | Primary patient reported outcome: intention to treat analysis of change in mean score for knee injury and osteoarthritis outcome subscale $\left(\mathrm{KOOS}_{4}\right)$ scores for pain, symptoms, function in sports and recreation, and knee related quality of life in exercise therapy group and arthroscopic partial meniscectomy group, from baseline to three month, 12 month, and two year follow-ups. Whiskers represent $95 \%$ confidence intervals

points (21.6 to 29.0) in the exercise group and 24.4 points (20.7 to 28.0) in the meniscectomy group (fig 2). Likewise, there were no clinically relevant differences between groups in $\mathrm{KOOS}_{4}$ score from baseline to follow-ups at 3 and 12 months (fig 2).

The results of per protocol and as treated analyses of between group differences in mean change from baseline to two year follow-up in $\mathrm{KOOS}_{4}$ score were similar to those of the intention to treat analysis; the difference in the per protocol analysis was 2.2 points ( -3.7 to 8.0; $\mathrm{P}=0.47)$ and in the as treated analysis was 2.0 points ( -4.1 to $8.1 ; \mathrm{P}=0.52$ ), both in favour of the exercise group (see supplementary table S1). Supplementary figure S5 shows the scores for the 13 participants who crossed over.

Sixty two participants in the exercise group and 64 in the meniscectomy group were included in the intention to treat analysis (fig 1). Both the per protocol and the as treated analysis included 34 participants in the exercise group, and 58 and 70 participants in the meniscectomy group, respectively (see supplementary figure S3).

For ease of interpretation, we also compared the proportion of participants reporting a clinically relevant

\footnotetext{
Muscle strength at 3 months Peak torque flexion ( $\mathrm{Nm})$ Total work flexion (J)

Peak torque extension ( $\mathrm{Nm}$ ) Total work extension (J) Muscle strength at $\mathbf{1 2}$ months Peak torque flexion $(\mathrm{Nm})$ Total work flexion (J) Peak torque extension ( $\mathrm{Nm}$ ) Total work extension (J)
}

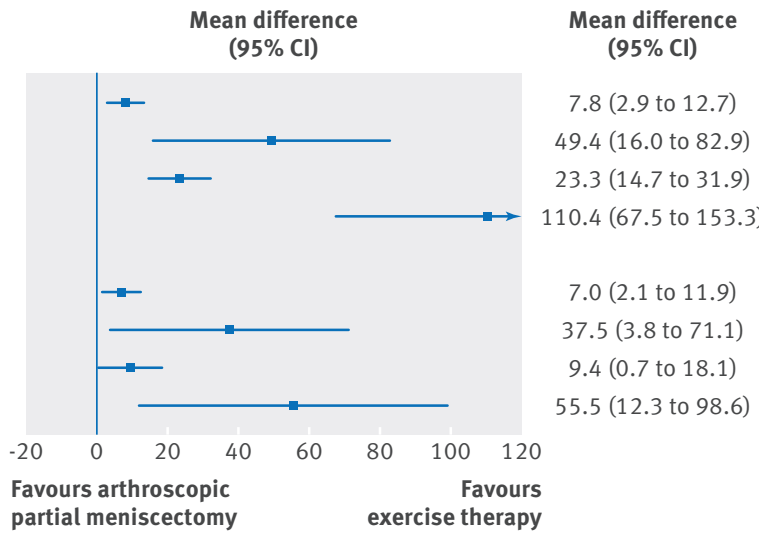

Fig 3 | Forest plots of intention to treat analyses of differences between groups in thigh muscle strength (peak torque ( $\mathrm{Nm}$ ) and total work (J) for knee extension and knee flexion, respectively) at three (primary endpoint) and 12 months improvement in $\mathrm{KOOS}_{4}$ at two years. For the intention to treat population, $80 \%$ in the exercise group and $81 \%$ in the meniscectomy group improved more than 10.1 points, with little difference in the per protocol $(81 \%$ and $79 \%$, respectively) and as treated populations $(81 \%$ and $79 \%$, respectively).

The exercise group had significantly greater improvement in all muscle strength variables at three months ( $\mathrm{P} \leq 0.004)$ (fig 3). Results were similar for the per protocol and as treated analyses (see supplementary table S2).

\section{Secondary outcomes}

Figures 3-5 show the results of the secondary outcomes at three months, 12 months, and two years (also see supplementary tables S1 and S2). From baseline to two year follow-up, the exercise group had a 5.3 point statistically significant but clinically insignificant greater improvement in scores on the KOOS subscale for symptoms than the meniscectomy group (95\% confidence interval 0.5 to $10.2 ; \mathrm{P}=0.03$ ). The study specific and subscale specific cut-offs for interpretation of clinically relevant differences were: 7.4 for pain, 8.4 for symptoms, 4.1 for activities of daily living, 10.9 for function in sport and recreation, and 13.6 for knee related quality of life. Clinically comparable improvements were found for all five KOOS subscales at all time points, with the exception of 12 months, where the meniscectomy group reported significantly or clinically relevant better scores for knee related quality of life and function in sport and recreation (see supplementary figure S4). There were no clinically relevant differences between the groups in SF-36. Results for per protocol and as treated analyses were similar to the intention to treat analyses (see supplementary tables S1 and S2).

The exercise group also had significantly greater improvement in all muscle strength tests at the 12 month follow-up $(\mathrm{P}<0.03)$. The exercise group had significantly greater improvement in the $6 \mathrm{~m}$ timed hop test at three months $(\mathrm{P}=0.02)$ and 12 months $(\mathrm{P}=0.04)$, but not in the one leg hop test or the knee bend test (fig 4). Results for per protocol and as treated analyses of the knee bend test were in favour of the exercise group at three months (see supplementary table S2).

\section{Harms}

From baseline to the two year follow-up, no serious adverse events were recorded in either group. During the same period, $23 \%$ of the participants in each group experienced pain, swelling, instability, stiffness, or decreased range of motion in the index knee that was serious enough to seek consultation. Similar symptoms in the contralateral knee were experienced by $21 \%$ of participants in the exercise group and $14 \%$ in the meniscectomy group.

\section{Discussion}

Supervised exercise therapy showed positive effects over arthroscopic partial meniscectomy in improving thigh muscle strength, at least in the short term, but not in patient reported outcomes, where the groups reported clinically comparable improvements at two 


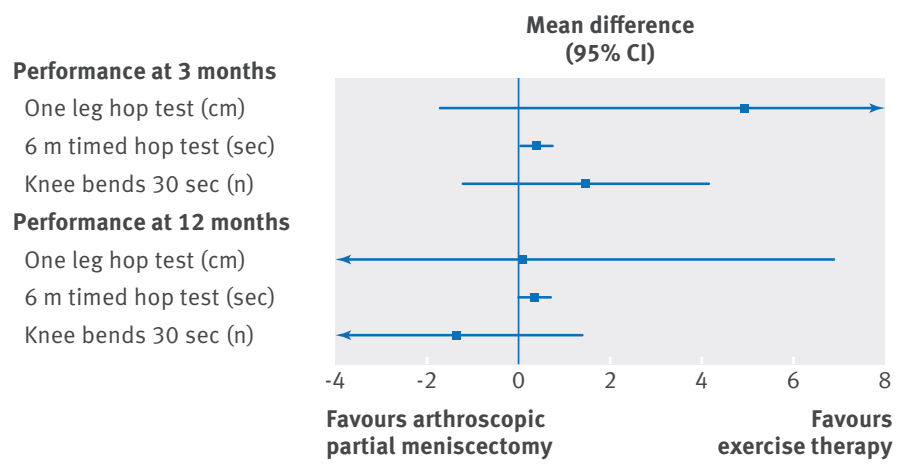

Fig 4 | Lower extremity performance tests: one leg hop test (cm), $6 \mathrm{~m}$ timed hop test (sec), and number of knee bends in 30 seconds ( $n$ ) at three and 12 months. Whiskers represent

$95 \%$ confidence intervals
Mean difference

$(95 \% \mathrm{Cl})$

$4.9(-1.7$ to 11.6$)$

0.4 (0.1 to 0.7$)$

$1.5(-1.2$ to 4.1$)$

$0.1(-6.7$ to 6.9$)$

0.4 (0.0 to 0.7$)$

$-1.3(-4.1$ to 1.4$)$

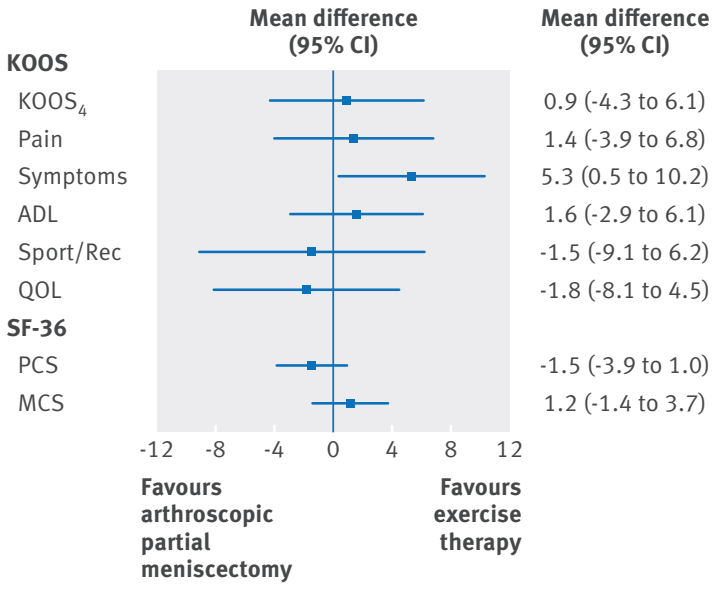

Fig 5 | Forest plots showing intention to treat analyses of between group differences in changes in primary patient reported outcome (mean score for knee injury and osteoarthritis outcome score (KOOS) subscales for pain, other symptoms, function in sport and recreation, and knee related quality of life $\left(\mathrm{KOOS}_{4}\right)$ ), and secondary outcomes for KOOS subscales and SF-36 physical component summary (PCS) and mental component summary (MCS) from baseline to two year follow-up. Whiskers represent $95 \%$ confidence intervals. $\mathrm{QOL}=$ quality of life; $A D L=a c t i v i t i e s$ of daily living

years. Our findings confirm previous studies evaluating the patient reported effect of surgery in addition to exercise compared with exercise alone. ${ }^{9-11} 13$ In our study, only $4 \%$ of participants had definitive radiographic evidence of osteoarthritis. Thus, our study extends previous findings to patients with early or no radiographic evidence of osteoarthritis.

Patient reported secondary outcomes confirmed no clinically relevant differences between groups at two years, with the exception of symptoms as measured on the knee injury and osteoarthritis outcome score (KOOS) scale, where the exercise group reported significantly fewer knee symptoms such as swelling, mechanical problems, and restricted range of motion.

One study reported an 8 point and 6 point significantly better outcome from surgery at three and 12 months, respectively, as evaluated using the KOOS subscale for pain. ${ }^{8}$ In the current study, at 12 months it could not be excluded that the surgically treated group reported a better outcome when evaluated using two other KOOS subscales: knee related quality of life and function in sport and recreation. This finding was, however, not present at three months or maintained at two years, suggesting it was of short duration.

\section{Strengths and limitations of this study}

Patient reported outcomes are prone to placebo effects. ${ }^{31}$ Although placebo effects are greater after invasive interventions such as surgery, ${ }^{31} 32$ they are also present from non-invasive passive treatments such as inactive ultrasonography and inert gel. ${ }^{33}$ In exercise studies, where the patient, not the therapist, performs the intervention, placebo effects are less obvious, although attention effects cannot be excluded. Objective outcomes are less prone to placebo effects, ${ }^{31}$ and a valuable addition for interpretation of clinical trials comparing surgical interventions with non-surgical interventions. Although we found no significant differences between treatments when evaluated using patient reported outcomes, muscle strength was significantly more improved in the supervised exercise group, directly after the intervention at both three months and 12 months. We cannot exclude the possibility that the greater placebo effect from surgery on patient reported outcomes masks a "real" difference in treatment between groups. It is a limitation of the study that we did not include a sham surgery group, which would have been needed to disentangle these mechanisms.

We used muscle strength testing as an objective outcome. Although muscle strength was immediately increased and maintained at 12 months in the exercise group, it was reduced at three months and only slightly better than at baseline at 12 months in the surgical group. It is reasonable to suggest that this strengthening effect of exercise therapy was maintained during the next year, and that measuring strength and functional performance at 12 months instead of two years as planned a priori is not supposed to have an influence on the results of this trial. Muscle strength is important for physical function, but interestingly the significantly greater improvement in muscle strength in the exercise group did not translate into consistently better functional performance. Knee extensor weakness is a risk factor for osteoarthritis, ${ }^{34}$ and longer term follow-up studies will show if exercise therapy and thigh muscle strength have the potential to mediate the high risk of radiographic osteoarthritis seen in patients with degenerative meniscal tears treated with arthroscopic partial meniscectomy.

The strengths of this study are the randomised controlled trial design, the multiple assessments, the high rate of participation in the two year follow-up, the use of valid and reliable patient reported outcomes, as well as inclusion of tests for muscle strength and performance ${ }^{2122}$ and the blinding of the assessors.

In addition to the lack of a sham surgery group, a limitation of comparing surgical with non-surgical treatment is the possibility of crossover from the non-surgical group to the surgical group. Crossover in 
our study was based on a clinical evaluation by the orthopaedic surgeon, initiated by either the participant or the physiotherapist. We failed to apply a stricter approach to determining crossover, such as requiring the evaluation of the patient's symptoms and knee related quality of life with a questionnaire that had a preset cut-off score.

All analyses were adjusted for baseline values. This is important because the participants in the meniscectomy group had somewhat better KOOS scores at baseline. In addition, the participants were slightly younger, had a lower body mass index, and reported knee pain for a shorter time than the participants randomised to exercise therapy. Their better baseline status may have provided participants in the meniscectomy group with an advantage, and, if anything, better results at follow-ups would be expected. This, however, was not the case.

Owing to slow patient flow, the recruitment process was taken over from participant number 54 by a hospital with a higher patient volume. To ensure consistency of recruiting procedures, the two recruiters participated in the first six recruiting sessions at the second hospital, and protocols and procedures were thoroughly discussed. Likewise, the surgical procedures at both hospitals were discussed among the participating surgeons to ensure consistency of protocols and procedures. Importantly, as there was no recruitment in parallel at the two hospitals, participants randomised to either treatment at a given time were drawn from the same pool of patients. Participant characteristics did not differ between those included at both sites. For geographical reasons, participants recruited at the second hospital and allocated to exercise therapy were treated at the second physiotherapy clinic. Multiple familiarisation sessions were held to ensure that exercise protocols were similar at both clinics.

\section{Comparison with other studies}

Compared with previous studies, our participants were younger (mean age 49.6 years), ${ }^{8-13}$ of a lower body mass index (26.2), ${ }^{112}$ and had fewer radiographic evidence of changes (only 4\% had definitive osteoarthritis). 811

Arthroscopic surgery of the knee is considered low risk surgery, the most common serious adverse event being deep vein thrombosis, with 4.13 (95\% confidence interval 1.78 to 9.60 ) events per 1000 procedures, followed by infection, pulmonary embolism, and death. ${ }^{7}$ No serious adverse events occurred in either treatment group in our study, indicating that in this comparatively younger, lower body mass, and more active population, both treatments were equally safe. A limitation is that our sample was too small to enable serious adverse events to be reliably detected.

\section{Conclusions and policy implications}

The observed difference in treatment effect was minute after two years' follow-up, and the trial's inferential uncertainty, as shown by the $95 \%$ confidence limits, was sufficiently small to exclude clinically relevant differences. Supervised exercise therapy showed positive effects over surgery in improving thigh muscle strength, at least in the short term. Nineteen per cent of participants allocated to exercise therapy crossed over to surgery during the two year follow-up, with no additional benefit. No serious adverse events occurred in either group during the two year follow-up. Our results should encourage clinicians and middle aged patients with degenerative meniscal tear and no radiographic evidence of osteoarthritis to consider supervised structured exercise therapy as a treatment option.

We thank the patients for their participation; research coordinators Kristin Bølstad and Emilie Jul-Larsen for the organisation of the participants; physiotherapists Marte Lund, Karin Rydevik, and Christian Vilming for assistance with data collection; the Norwegian Sports Medicine Clinic (NIMI), Oslo, Norway, for supporting the Norwegian Research Center for Active Rehabilitation (NAR) with rehabilitation facilities and research staff (NAR is a collaboration between the Norwegian School of Sports Sciences, Department of Orthopaedic Surgery, Oslo University Hospital, and NIMI); and the Department of Orthopaedic Surgery, Oslo University Hospital and the Department of Orthopaedic Surgery, Martina Hansens Hospital, Bærum, for accessibility to the outpatient and surgical clinics.

Contributors: EMR, MAR, and SS conceived and designed the study. SS, LE, and NJK collected the data. SS trained most participants. LE and NJK operated on most participants. NJK and EMR drafted the article. Jonas Ranstam performed the statistical analyses. All authors participated in the analysis and interpretation of the data, revision of the article, and final approval of the version to be published. EMR is the guarantor. All authors had full access to all of the data including statistical reports and tables in the study and take responsibility for the integrity of the data and the accuracy of the data analysis.

Funding: This study was funded by Sophies Minde Ortopedi AS, Swedish Rheumatism Association, Swedish Scientific Council, Region of Southern Denmark, Danish Rheumatism Association, and the Health Region of South-East Norway. The researchers were independent from the funder.

Competing interests: All authors have completed the ICMJE uniform disclosure form at www.icmje.org/coi_disclosure.pdf (available on request from the corresponding author) and declare: no support from any company for the submitted work; no relationships with any company that might have an interest in the submitted work in the previous three years; their spouses, partners, or children have no financial relationships that may be relevant to the submitted work; and they have no non-financial interests that may be relevant to the submitted work.

Ethical approval: This study was approved by the regional ethics committee of the Health Region of South-East Norway (reference No 2009/230).

Data sharing: Anonymised data will be shared on reasonable request.

Transparency: The lead author (N/K) affirms that the manuscript is an honest, accurate, and transparent account of the study being reported; that no important aspects of the study have been omitted; and that any discrepancies from the study as planned have been explained. Data sharing: we agree to share anonymised data upon reasonable request.

This is an Open Access article distributed in accordance with the Creative Commons Attribution Non Commercial (CC BY-NC 3.0) license, which permits others to distribute, remix, adapt, build upon this work non-commercially, and license their derivative works on different terms, provided the original work is properly cited and the use is non-commercial. See: http://creativecommons.org/licenses/ by-nc/3.0/.

1 Abrams GD, Frank RM, Gupta AK, Harris JD, McCormick FM, Cole BJ. Trends in meniscus repair and meniscectomy in the United States, 2005-2011. Am J Sports Med 2013;41:2333-9. doi:10.1177/0363546513495641.

2 Lohmander LS, Englund PM, Dahl LL, Roos EM. The long-term consequence of anterior cruciate ligament and meniscus injuries: osteoarthritis. Am J Sports Med 2007;35:1756-69. doi:10.1177/0363546507307396.

3 Reigstad O, Grimsgaard C. Complications in knee arthroscopy. Knee Surg Sports Traumatol Arthrosc 2006;14:473-7. doi:10.1007/ s00167-005-0694-x. 
4 Thorlund JB, Hare KB, Lohmander LS. Large increase in arthroscopic meniscus surgery in the middle-aged and older population in Denmark from 2000 to 2011. Acta Orthop 2014:85:287-92. doi:10.3109/17453674.2014.919558.

5 Englund $M$. The role of the meniscus in osteoarthritis genesis. Med Clin North Am 2009;93:37-43, x. doi:10.1016/j.mcna.2008.08.005.

6 Englund M, Roos EM, Roos HP, Lohmander LS. Patient-relevant outcomes fourteen years after meniscectomy: influence of type of meniscal tear and size of resection. Rheumatology (Oxford) 2001:40:631-9. doi:10.1093/rheumatology/40.6.631

7 Thorlund JB, Juhl CB, Roos EM, Lohmander LS. Arthroscopic surgery for degenerative knee: systematic review and meta-analysis of benefits and harms. Br J Sports Med 2015;49:1229-35. doi:10.1136/ bjsports-2015-h2747rep.

8 Gauffin H, Tagesson S, Meunier A, Magnusson H, Kvist J. Knee arthroscopic surgery is beneficial to middle-aged patients with meniscal symptoms: a prospective, randomised, single-blinded study. Osteoarthritis Cartilage 2014;22:1808-16. doi:10.1016/j.joca.2014. 07.017.

9 Herrlin S, Hållander M, Wange P, Weidenhielm L, Werner S. Arthroscopic or conservative treatment of degenerative medial meniscal tears: a prospective randomised trial. Knee Surg Sports Traumatol Arthrosc 2007;15:393-401. doi:10.1007/s00167-006-0243-2.

10 Herrlin SV, Wange PO, Lapidus G, Hållander M, Werner S, Weidenhielm L. Is arthroscopic surgery beneficial in treating non-traumatic, degenerative medial meniscal tears? A five year follow-up. Knee Surg Sports Traumatol Arthrosc 2013;21:358-64. doi:10.1007/ s00167-012-1960-3.

11 Katz JN, Brophy RH, Chaisson CE, et al. Surgery versus physical therapy for a meniscal tear and osteoarthritis. N Engl J Med 2013;368:1675-84. doi:10.1056/NEIMoa1301408.

12 Sihvonen R, Paavola M, Malmivaara A, et al. Finnish Degenerative Meniscal Lesion Study (FIDELITY) Group. Arthroscopic partial meniscectomy versus sham surgery for a degenerative meniscal tear. N Engl J Med 2013;369:2515-24. doi:10.1056/NEJMoa1305189.

13 Yim JH, Seon JK, Song EK, et al. A comparative study of meniscectomy and nonoperative treatment for degenerative horizontal tears of the medial meniscus. Am J Sports Med 2013;41:1565-70. doi:10.1177/ 0363546513488518.

14 Østerås H, Østerås B, Torstensen TA. Medical exercise therapy, and not arthroscopic surgery, resulted in decreased depression and anxiety in patients with degenerative meniscus injury. J Bodyw Mov Ther 2012;16:456-63. doi:10.1016/j.jbmt.2012.04.003.

15 Stensrud S, Risberg MA, Roos EM. Effect of exercise therapy compared with arthroscopic surgery on knee muscle strength and functional performance in middle-aged patients with degenerative meniscus tears: a 3-mo follow-up of a randomized controlled trial. Am J Phys Med Rehabil 2015:94:460-73. doi:10.1097/PHM.0000000000000209.

16 Hall M, Juhl CB, Lund H, Thorlund JB. Knee Extensor Muscle Strength in Middle-Aged and Older Individuals Undergoing Arthroscopic Partial Meniscectomy: A Systematic Review and Meta-Analysis. Arthritis Care Res (Hoboken) 2015;67:1289-96. doi:10.1002/acr.22581.

17 Kellgren JH, Lawrence JS. Radiological assessment of osteo-arthrosis. Ann Rheum Dis 1957:16:494-502. doi:10.1136/ard.16.4.494.

18 Kothari M, Guermazi A, von Ingersleben G, et al. Fixed-flexion radiography of the knee provides reproducible joint space width measurements in osteoarthritis. Eur Radiol 2004;14:1568-73. doi:10.1007/s00330-004-2312-6
19 Crues JV 3rd, Mink J, Levy TL, Lotysch M, Stoller DW. Meniscal tears of the knee: accuracy of MR imaging. Radiology 1987;164:445-8. doi:10.1148/radiology.164.2.3602385.

20 Stensrud S, Roos EM, Risberg MA. A 12-week exercise therapy program in middle-aged patients with degenerative meniscus tears: a case series with 1-year follow-up. J Orthop Sports Phys Ther 2012;42:919-31. doi:10.2519/jospt.2012.4165.

21 Roos EM, Lohmander LS. The Knee injury and Osteoarthritis Outcome Score (KOOS): from joint injury to osteoarthritis. Health Qual Life Outcomes 2003:1:64. doi:10.1186/1477-7525-1-64

22 Roos EM, Roos HP, Ekdahl C, Lohmander LS. Knee injury and Osteoarthritis Outcome Score (KOOS)-validation of a Swedish version. Scand J Med Sci Sports 1998;8:439-48. doi:10.1111/j.1600-0838.1998.tb00465.x.

23 Kamper SJ, Maher CG, Mackay G. Global rating of change scales: a review of strengths and weaknesses and considerations for design. Man Manip Ther 2009:17:163-70. doi:10.1179/jmt.2009.17.3.163.

24 Drouin JM, Valovich-mcLeod TC, Shultz SJ, Gansneder BM, Perrin DH. Reliability and validity of the Biodex system 3 pro isokinetic dynamometer velocity, torque and position measurements. Eur J Appl Physiol 2004:91:22-9. doi:10.1007/s00421-003-0933-0.

25 Ware JE Jr, Sherbourne CD. The MOS 36-item short-form health survey (SF-36). I. Conceptual framework and item selection. Med Care 1992:30:473-83 doi:10.1097/00005650-199206000-00002.

26 Ross MD, Langford B, Whelan PJ. Test-retest reliability of 4 single-leg horizontal hop tests. J Strength Cond Res 2002;16:617-22.

27 Bolgla LA, Keskula DR. Reliability of lower extremity functional performance tests. J Orthop Sports Phys Ther 1997;26:138-42. doi:10.2519/jospt.1997.26.3.138

28 Bremander AB, Dahl LL, Roos EM. Validity and reliability of functional performance tests in meniscectomized patients with or without knee osteoarthritis. Scand I Med Sci Sports 2007:17:120-7.

29 Stensrud S, Risberg MA, Roos EM. Knee function and knee muscle strength in middle-aged patients with degenerative meniscal tears eligible for arthroscopic partial meniscectomy. Br J Sports Med 2014:48:784-8. doi:10.1136/bjsports-2012-091540.

30 Satterthwaite FE. An approximate distribution of estimates of variance components. Biometrics 1946;2:110-4. doi:10.2307/3002019.

31 Hróbjartsson A, Gøtzsche PC. Placebo interventions for all clinical conditions. Cochrane Database Syst Rev 2010;(1):CD003974

32 Zou K, Wong J, Abdullah N, et al. Examination of overall treatment effect and the proportion attributable to contextual effect in osteoarthritis: meta-analysis of randomised controlled trials. Ann Rheum Dis 2016;annrheumdis-2015-208387. doi:10.1136/ annrheumdis-2015-208387.

33 Hinman RS, McCrory P, Pirotta M, et al. Acupuncture for chronic knee pain: a randomized clinical trial. JAMA 2014;312:1313-22 doi:10.1001/jama.2014.12660.

34 Øiestad BE, Juhl CB, Eitzen I, Thorlund JB. Knee extensor muscle weakness is a risk factor for development of knee osteoarthritis. A systematic review and meta-analysis. Osteoarthritis Cartilage 2015;23:171-7. doi:10.1016/j.joca.2014.10.008.

\section{Web supplement: supplementary information}

